\title{
Automatic Multiresolution Age-related Macular Degeneration Detection from Fundus Images
}

\author{
Mickaël Garnier ${ }^{1,2}$, Thomas Hurtut ${ }^{1,2}$, Houssem Ben Tahar $^{3}$, and Farida Cheriet ${ }^{2}$ \\ ${ }^{1}$ LIPADE, University of Paris Descartes, France \\ ${ }^{2}$ École Polytechnique de Montréal, Canada \\ ${ }^{3}$ DIAGNOS inc., Canada
}

\begin{abstract}
Age-related Macular Degeneration (AMD) is a leading cause of legal blindness. As the disease progress, visual loss occurs rapidly, therefore early diagnosis is required for timely treatment. Automatic, fast and robust screening of this widespread disease should allow an early detection. Most of the automatic diagnosis methods in the literature are based on a complex segmentation of the drusen, targeting a specific symptom of the disease. In this paper, we present a preliminary study for AMD detection from color fundus photographs using a multiresolution texture analysis. We analyze the texture at several scales by using a wavelet decomposition in order to identify all the relevant texture patterns. Textural information is captured using both the sign and magnitude components of the completed model of Local Binary Patterns. An image is finally described with the textural pattern distributions of the wavelet coefficient images obtained at each level of decomposition. We use a Linear Discriminant Analysis for feature dimension reduction, to avoid the curse of dimensionality problem, and image classification. Experiments were conducted on a dataset containing 45 images ( 23 healthy and 22 diseased) of variable quality and captured by different cameras. Our method achieved a recognition rate of $93.3 \%$, with a specificity of $95.5 \%$ and a sensitivity of $91.3 \%$. This approach shows promising results at low costs that in agreement with medical experts as well as robustness to both image quality and fundus camera model.
\end{abstract}

Keywords: Computer-aided diagnosis, Age-related Macular Degeneration, Multiresolution, Texture Analysis, Linear Discriminant Analysis

\section{INTRODUCTION}

Age-related Macular Degeneration (AMD) is one of the most common causes of legal blindness in the world, and the most important among the elder population of developed countries.1] At an advanced stage, AMD can quickly cause the loss of central vision. However, it can progress from an early stage without being noticed due to the lack of obvious visual changes. Therefore, early diagnosis is a major issue in order to be able to correctly treat the disease and avoid complications. There are two types of AMD, the wet and the dry but both are characterized by the presence of drusen, the symptomatic landmark of the disease.

Many imaging systems are used for eye diagnosis, the most noteworthy being color and fluorescein angiography fundus photography, and optical coherence tomography (OCT). To detect AMD, OCT presents the advantages of being able to easily show both the retina damages and the blood collection in the choroid, hence helps early diagnosis but is still expensive with a slow image acquisition. Angyography focuses on blood vessels through injection of contrast agent whereas color fundus photography capture both structural and vessels information. Color fundus images are also the most spread device because of its cost, its availability and its simplicity.

The automatic diagnosis of AMD is usually made based on the detection of drusen on fundus images ${ }^{[1[5]}$ although there is no medical consensus whether drusen always are a clue for early AMD, 6 and early microscopic drusen are hard to detect, even clinically ${ }^{7}$ Other symptoms of AMD include pigmentary changes ${ }^{5}$ as well as fluid leakage and fast ingrowth of vessels which are both due to choroidal neovascularization in the case of wet AMD. ${ }^{1}$ Many drusen detection algorithms exist in the literature. They are mostly based on first the localization of high intensity areas. Then several features such as

For further information send correspondence to:

Mickaël Garnier: E-mail: mgarnier@math-info.univ-paris5.fr, Telephone: (+33) 183945824 


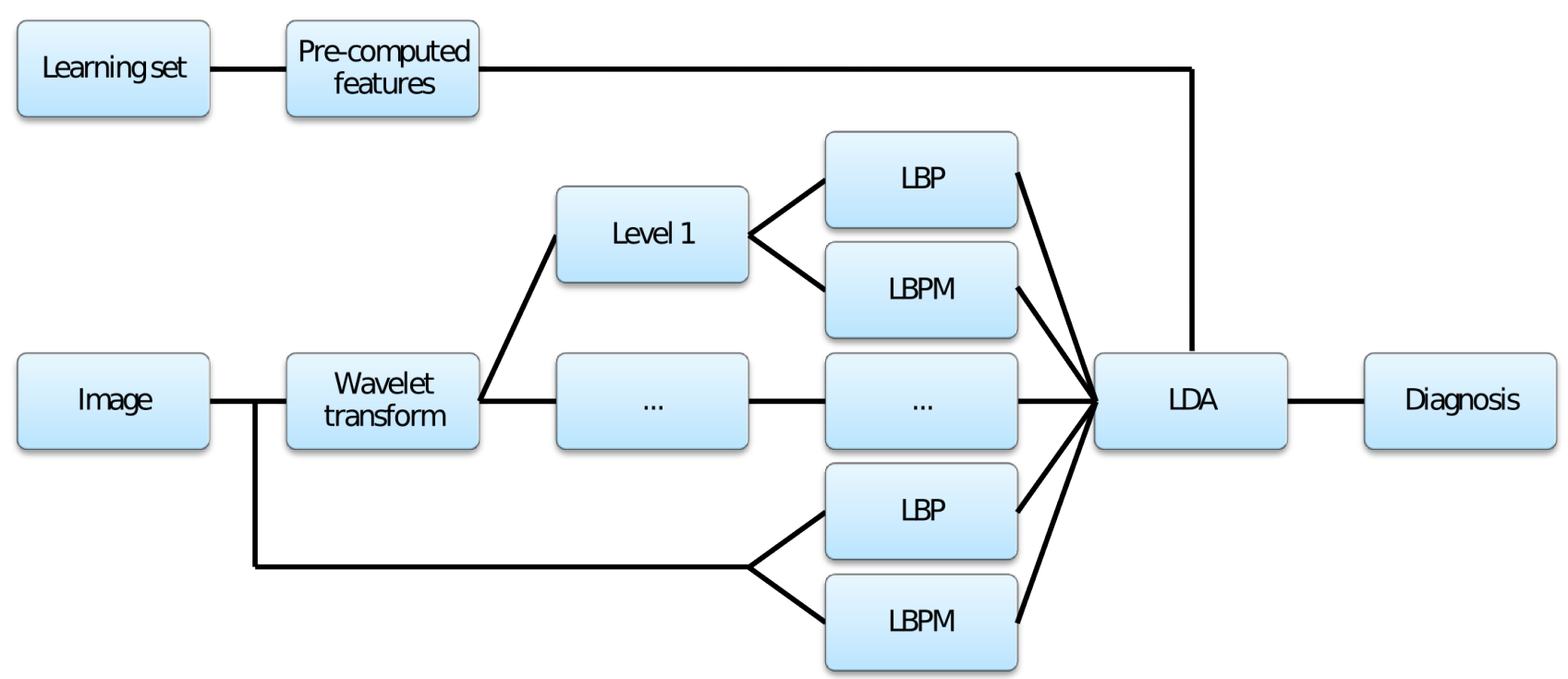

Figure 1. Overview of our method. An input image is decomposed with a wavelet transform. Every coefficient images of each level are then described using Local Binary Patterns features. Finally, a Linear Discriminant Analysis, previously trained on a learning set, is used to linearly classify the image as either negative or positive to the presence of AMD.

color, shape and neighborhood, are computed to measure a probability map for all the potential drusen. Classification is then made based on this probability map to discriminate diseased patient from healthy ones. These methods are unable to detect symptoms other than visible drusen and require adequate image quality.

Contrary to other AMD detection methods, our innovative approach aims at measuring all the relevant variations induced by the disease in fundus photographs. The idea is to be able to detect early stages of the disease without targeting any particular symptom. Therefore, the proposed method is based on a multiresolution texture analysis ensuring that all the relevant texture patterns are captured and taken into account.

\section{METHOD}

In order to analyze the texture information on several scales, we first need to access this multiresolution information. To do so, we uses a wavelet transform which also give us the possibility to study the high frequency information contained inside the image. All this information is then described using Local Binary Patterns based features. Since this method gathers a lot of information, we need to focus our description on the most relevant ones. Thus, a Linear Discriminant Analysis is used to favors the most discriminant features from a training dataset. Finally, the outcome of the LDA is used to linearly classify the image. A summary of our method is given in Figure 1.

\subsection{Multiresolution analysis}

The wavelet filter-bank is successfully used in multiresolution analysis. For every image, it gives both a scale invariant interpretation, thanks to the approximation coefficients, and an access to high frequency information through the detail coefficients. Our images are decomposed using the Lemarié's wavelet, which is frequently used to analyze the information content of images $\frac{[8}{[}$

$$
H(\omega)=\left[2(1-u)^{4} \times \frac{315-420 u+126 u^{2}-4 u^{3}}{315-420 v+126 v^{2}-4 v^{3}}\right]^{\frac{1}{2}}
$$

where:

$$
\begin{aligned}
u & =\sin ^{2}\left(\frac{1}{2} \omega\right) \\
v & =\sin ^{2}(\omega)
\end{aligned}
$$

We decompose our images up to four levels, giving us a set of 16 coefficient images in addition to the original image for textural features extraction. These 17 images are then used for textural information extraction. The decomposition of an 
healthy and an AMD afflicted images are shown in Figures 2 and 3 . These figures highlight the textural differences induced by the disease.
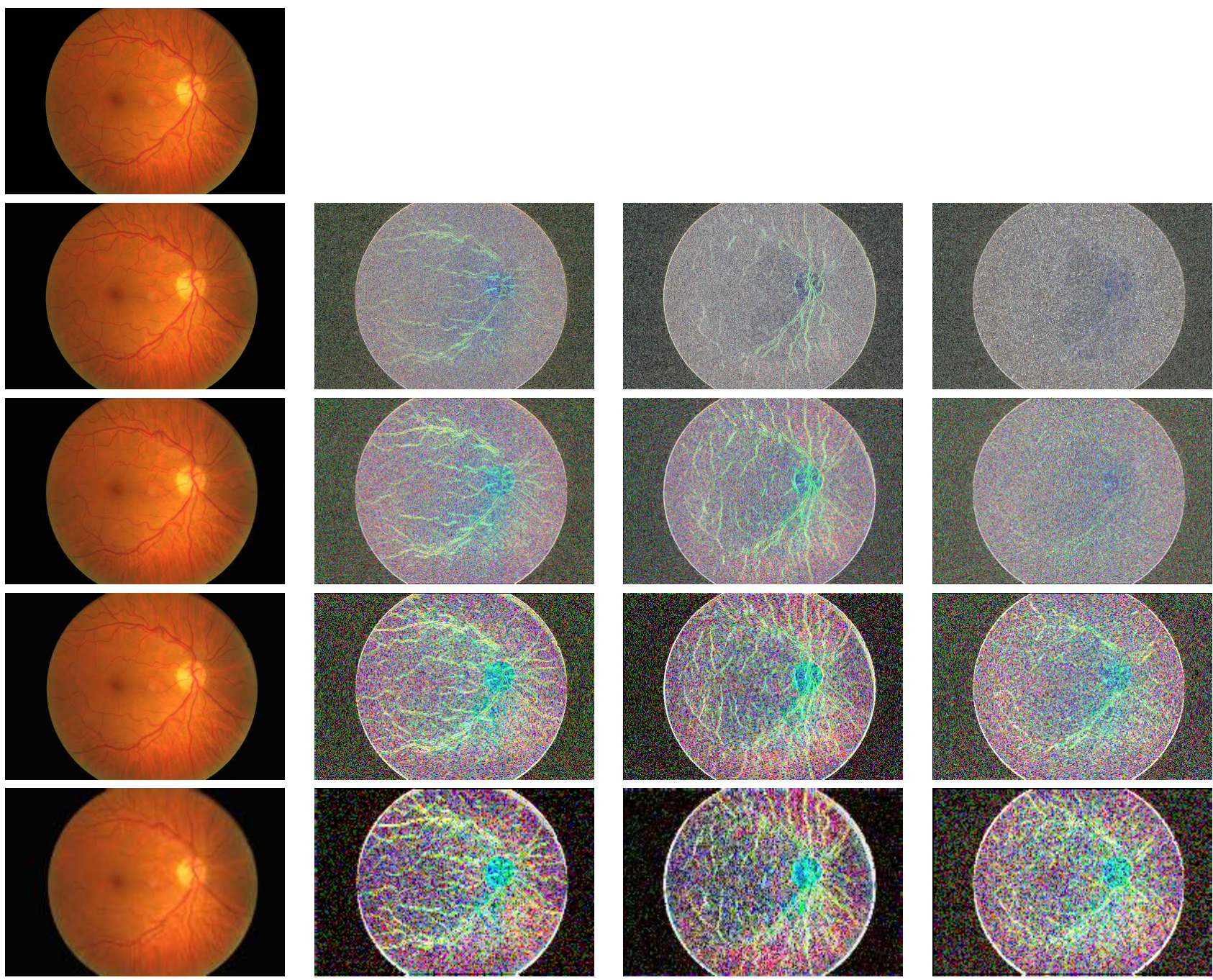

Figure 2. Wavelet decomposition on a healthy image from our database. Each row corresponds to a level of decomposition, the first column shows the approximation coefficient images, the second, third and fourth display respectively the horizontal, vertical and diagonal detail coefficient images. The first image is the original one. For a better visualization, the wavelet detail coefficient images have been processed with an histogram equalization.

\subsection{Textural features extraction}

Presented by Ojala et al., 9 the uniform Local Binary Patterns (LBP) have been widely used for texture analysis. Several applications such as face expression recognition, $, 10,11$ emphasize the efficiency of this descriptor. This method aims at measuring the occurrences of local textures primitives, the resulting feature being their distribution. Here, the textural information is extracted using the distributions of both the classical LBP and the magnitude (LBPM) component of the completed model of LBP, $\frac{12}{12}$ the two information being complementary.

The local texture pattern around a pixel $\left(x_{c}, y_{c}\right)$ of gray value $g_{c}$ is defined as:

$$
\begin{gathered}
L B P_{P, R}=\sum_{P=0}^{P-1} s\left(g_{p}-g_{c}\right) \times 2^{P} \\
s(x)=\left\{\begin{array}{ll}
1 & \text { if } x \geq 0 \\
0 & \text { otherwise }
\end{array}, \forall x \in \mathbf{Z}\right.
\end{gathered}
$$



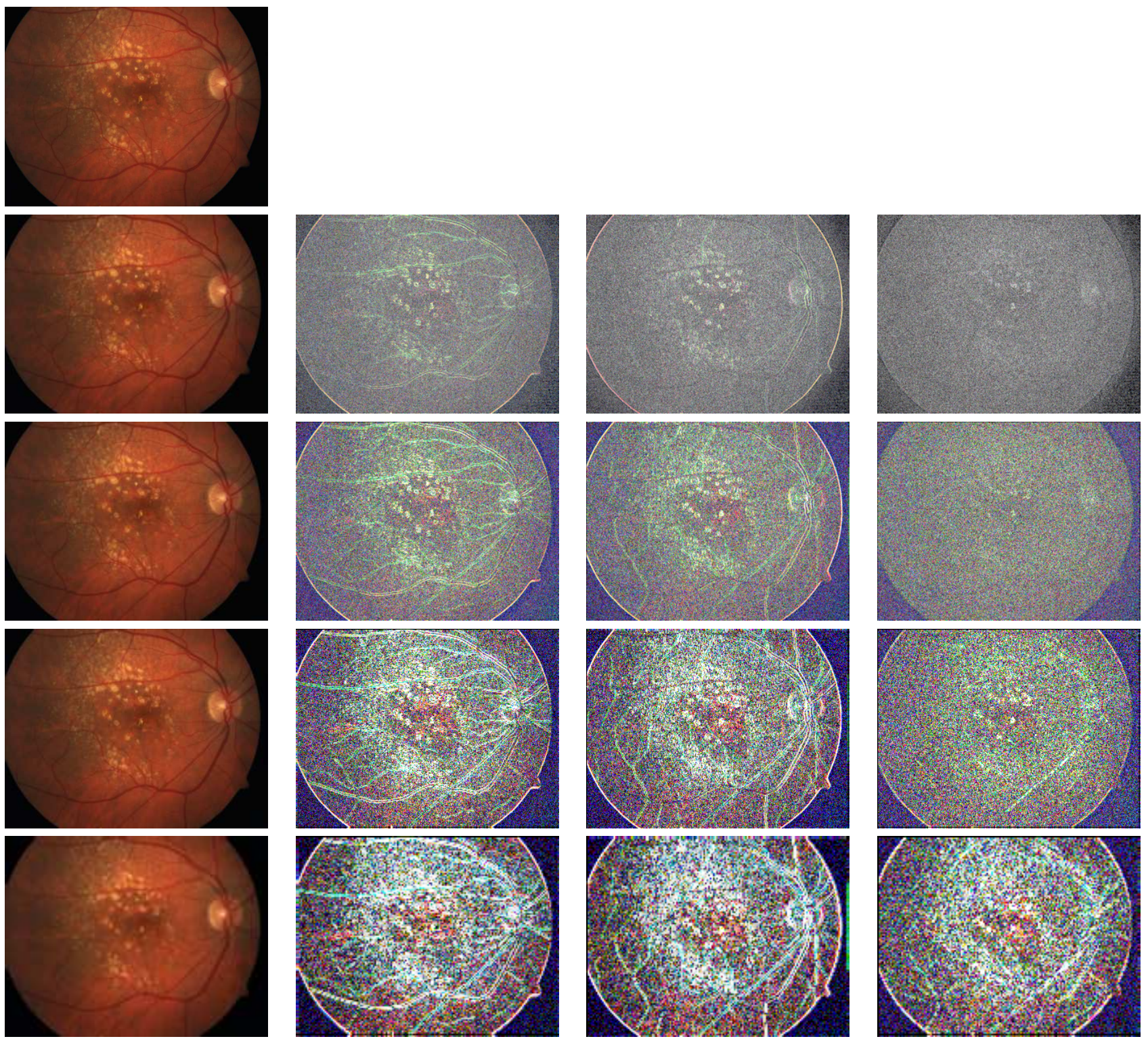

Figure 3. Wavelet decomposition on an AMD afflicted image from our database. Each row corresponds to a level of decomposition, the first column shows the approximation coefficient images, the second, third and fourth display respectively the horizontal, vertical and diagonal detail coefficient images. The first image is the original one. For a better visualization, the wavelet detail coefficient images have been processed with an histogram equalization. 
where $\left(g_{p}\right)_{\forall p \in\{0 . . P-1\}}$ correspond to the gray level values of equally spaced pixels $P$ on a circle of radius $R$ around the pixel $\left(x_{c}, y_{c}\right)$. To capture supplemental information we also measure the magnitude component from the completed LBP 12 defined as:

$$
\begin{aligned}
& \operatorname{LBP}_{P, R}=\sum_{p=0}^{P-1} t\left(g_{p}-g_{c}, \nu\right) \times 2^{P} \\
& t(x, \nu)=\left\{\begin{array}{ll}
1 & \text { if } x \geq \nu \\
0 & \text { otherwise }
\end{array}, \forall x \in \mathbf{Z}\right.
\end{aligned}
$$

where $\nu$ is a binarization threshold set to the mean of the $g_{p}-g_{c}$ values over the whole image.

We use the uniform LPB which reduces the feature dimension from $2^{P}$ to $P(p-1)+3$ and still captures the local image texture.

The LBP can be set to measure texture information at several scales thanks to the radius parameter. In this work, we fixed this parameter to $R=1$ and rely on the wavelet decomposition for the multiresolution information. The decomposition has the advantage of separating the information according to its frequency, thus allowing for a more complete description.

\subsection{Features dimension reduction and classification}

Finally, our images are described by the distributions of LBP and LBPM of every wavelet coefficient images, giving features with a high dimensionality: 2 histograms of 59 bins times 17 coefficients, for a final dimension of 2006. To avoid the curse of dimensionality problem and give more importance to relevant features, we apply a linear Discriminant Analysis (LDA) ${ }^{13}$ for feature dimension reduction. The LDA finds a linear combination of the features able to separates the two classes. It is trained on a learning set and then used to linearly classify our data. Due to the size of our dataset, we use a leave-one-out validation method, namely the learning set is composed of all the images except the one being tested.

\section{EXPERIMENTS}
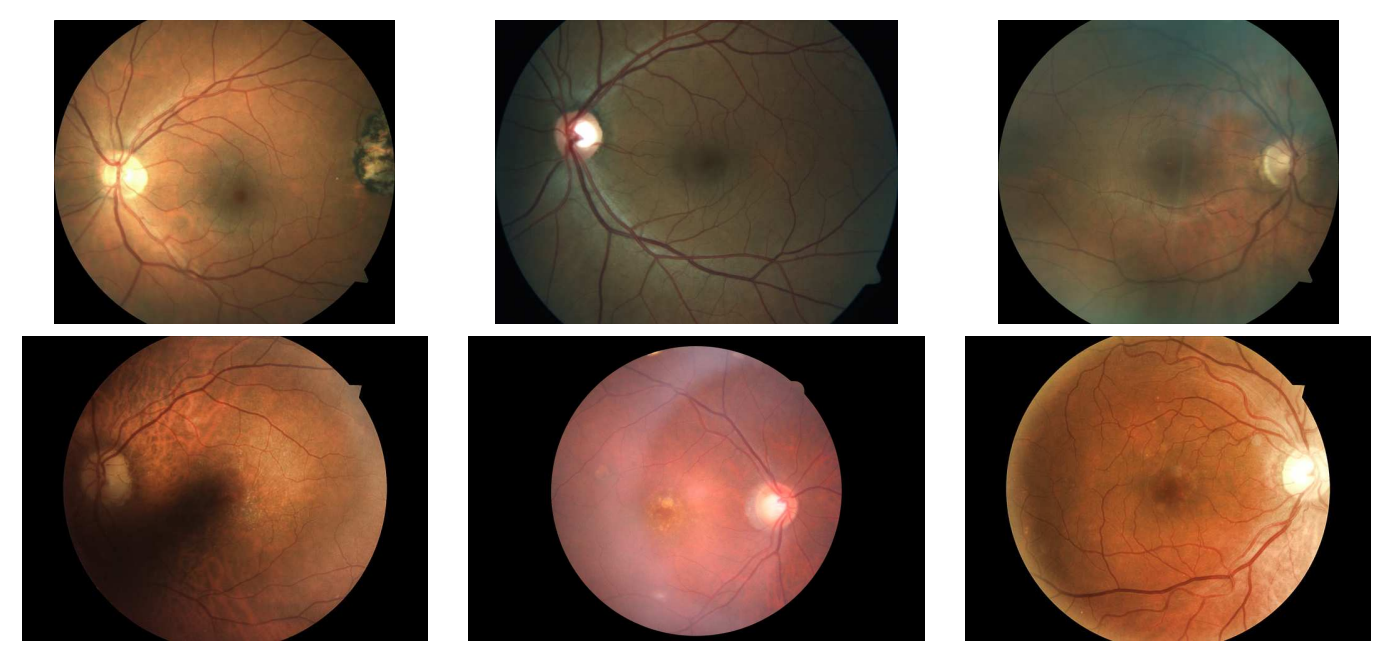

Figure 4. Sample images from our database showing its variability and diagnosis difficulties. Healthy images are displayed in the first row and AMD afflicted in the second row.

To asses the efficiency of our method a preliminary study was conducted on a private datase 17 classified by medical experts. This set is composed of 45 images, 22 afflicted with AMD and 23 healthy. The advantage of this dataset relies on the variability of the images, being captured by different cameras, presenting different ethnic groups and age categories and mainly offering different qualities, going from poor to very good due to illumination variations, blur and reflections. Some representative images are shown in Figure 4.

\footnotetext{
${ }^{1}$ Property of DIAGNOS inc.
} 
Our preliminary results are given in Table 1 . The proposed method achieves a good disease detection, with a recognition rate, a specificity and a sensitivity of respectively $93.3 \%, 95.5 \%$ and $91.3 \%$. These results also shows that our method is robust to image quality, a required quality for medical screenings.

These results underlines the efficiency of the proposed method to accurately measure and target the relevant textural information among all the wavelet coefficient images. It also shows that our method is robust to image quality and illumination invariant. Since no symptoms are specifically targeted, it is not limited to the presence of drusen and take other information into account.

Table 1. Confusion matrix obtained on our dataset. It shows a specificity of $95.5 \%$, a sensitivity of $91.3 \%$ and a recognition rate of $93.3 \%$ with only 3 misdiagnosed images.

\begin{tabular}{|c|c|c|}
\hline \multirow{3}{*}{ 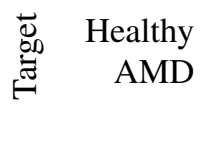 } & 21 & 2 \\
\hline & 1 & 21 \\
\hline & $\begin{array}{r}\text { Healthy } \\
\text { Out }\end{array}$ & AMD \\
\hline
\end{tabular}

\begin{tabular}{ccc}
\hline Specificity & Sensitivity & Recognition Rate \\
\hline $95.5 \%$ & $91.3 \%$ & $93.3 \%$ \\
\hline
\end{tabular}

\section{CONCLUSION}

This paper presents a method for AMD detection based on a multiresolution analysis of small texture elements. It shows both good detection results and robustness to variations related to image acquisition while avoiding targeting any specific symptoms. This data-driven approach does not require any structural segmentation or preprocessing in addition to be unsupervised, except for the classifier training. Therefore, this method is promising for clinical screenings as it should lead to a reliable and low-cost automated diagnosis system.

This preliminary investigation demonstrates the effectiveness of a multi-scale texture analysis for Age-related Macular Degeneration detection. It highlights the discriminative strength of the texture to diagnose this particular disease, which can be useful especially in early stages.

Besides performing an extensive validation of this method, the next step is to find a strategy to find out which wavelet coefficients are relevant concerning the AMD before feature dimension reduction. This might be done using a feature selection method. We think this should render the diagnosis robust to other diseases such as the common diabetic retinopathy. Such improvement should also make this method easily adaptable for other diseases detection.

\section{Acknowledgement}

This work was partially supported by a French National Research Agency project (SPIRIT \#11-JCJC-008-01) and a Canadian Natural Sciences and Engineering Research Council (NSERC) Collaborative Research and Development grant.

\section{REFERENCES}

[1] Abràmoff, M. D., Garvin, M. K., and Sonka, M., "Retinal imaging and image analysis," IEEE Reviews in Biomedical Engineering 3, 169-208 (2010).

[2] Mora, A. D., Vieira, P. M., Manivannan, A., and Fonseca, J. M., "Automated drusen detection in retinal images using analytical modelling algorithms," Biomedical engineering online 10(1), 59 (2011).

[3] Remeseiro, B., Barreira, N., Calvo, D., Ortega, M., and Penedo, M. G., "Automatic drusen detection from digital retinal images: Amd prevention," in [Computer Aided Systems Theory-EUROCAST 2009], 187-194, Springer (2009).

[4] Zheng, Y., Vanderbeek, B., Daniel, E., Stambolian, D., Maguire, M., Brainard, D., and Gee, J., "An automated drusen detection system for classifying age-related macular degeneration with color fundus photographs," in [10th International Symposium on Biomedical Imaging (ISBI)], IEEE (2013).

[5] van Grinsven, M. J., Lechanteur, Y. T., van de Ven, J. P., van Ginneken, B., Theelen, T., and Sánchez, C. I., "Automatic age-related macular degeneration detection and staging," in [SPIE Medical Imaging], 86700M-86700M, International Society for Optics and Photonics (2013).

[6] Jager, R. D., Mieler, W. F., and Miller, J. W., "Age-related macular degeneration," New England Journal of Medicine 358(24), 2606-2617 (2008). 
[7] Sarks, S., Arnold, J., Killingsworth, M., and Sarks, J., "Early drusen formation in the normal and aging eye and their relation to age related maculopathy: a clinicopathological study," British Journal of Ophthalmology 83(3), 358-368 (1999).

[8] Mallat, S. G., "A theory for multiresolution signal decomposition: the wavelet representation," IEEE Transactions on Pattern Analysis and Machine Intelligence 11(7), 674-693 (1989).

[9] Ojala, T., Pietikainen, M., and Maenpaa, T., "Multiresolution gray-scale and rotation invariant texture classification with local binary patterns," IEEE Transactions on Pattern Analysis and Machine Intelligence 24(7), 971-987 (2002).

[10] Guo, Z., Zhang, L., Zhang, D., and Mou, X., "Hierarchical multiscale lbp for face and palmprint recognition," in [17th IEEE International Conference on Image Processing (ICIP)], 4521-4524, IEEE (2010).

[11] Shan, C., Gong, S., and McOwan, P. W., "Facial expression recognition based on local binary patterns: A comprehensive study," Image and Vision Computing 27(6), 803-816 (2009).

[12] Zhenhua, G., Zhang, L., and Zhang, D., "A completed modeling of local binary pattern operator for texture classification," IEEE Transactions on Image Processing 19(6), 1657-1663 (2010).

[13] Fukunaga, K., [Introduction to statistical pattern recognition], Access Online via Elsevier (1990). 